\title{
Safety and efficacy of flexible and semi-rigid ureteroscopy with laser lithotripsy for the management of ureteral calculi in pregnancy
}

\author{
Aso Omer Rashid ${ }^{1 *}$ (I) and Rezhin Yaseen Abdala²
}

\begin{abstract}
Background: Symptomatic ureteric stone during pregnancy can present a clinical challenge because of potential risks to both the mother and foetus. Ureteroscopy with laser and stone basket extraction represents an emerging strategy for definitive stone management in pregnancy, with minor complications. We aimed to evaluate the safety and efficacy of ureteroscopy with auxiliary procedures as a primary treatment for pregnant women with symptomatic ureteric stones who have failed conservative management.

Methods: A prospective analysis was conducted in the Urology Department of Sulaymaniyah Teaching Hospital from June 2017 to November 2019 to evaluate pregnant patients who were treated with ureteroscopy and holmium laser lithotripsy for symptomatic ureteric stone.

Results: Twenty-six pregnant women aged between 18 and 34 years presented with renal colic (22 patients, 84.61\%), severe hydronephrosis (3 patients, 11.53\%), and progressive hydronephrosis (1 patient, 3.8\%), suggesting ureteric stones. The diagnosis was established by abdominal ultrasonography. The mean stone size was $7.38 \mathrm{~mm}$, ranging from 6-12 mm. The stones were located in the proximal ureter $(n=6,23.1 \%)$, middle ureter $(n=0)$, and distal ureter $(n=20,76.9 \%)$. Complete stone fragmentation was achieved in all patients. The overall procedure success rate was $87 \%$, the stone-free rate was 23 out of 26 cases $(88.46 \%)$, and no major obstetric or urologic complications were encountered.

Conclusions: Ureteric stone in pregnancy requires a high index of suspicion for a prompt and correct diagnosis. Ureteroscopy: flexible or semirigid endoscopy combined with holmium: YAG laser lithotripsy or with stone extraction are a preferred modality for the definitive treatment of symptomatic ureteric stone in pregnancy.
\end{abstract}

Keywords: Endourology, Ureteric stones, Pregnancy, Laser lithotripsy, Safety, Efficacy

\section{Background}

Urinary stone disease is found in 1:500 pregnant women, with no apparent differences in prevalence found [1]. Pregnancy is not a significant risk factor for ureteric stone, but the diagnosis is challenging and has management pitfalls [2]. The increasing progesterone levels

\footnotetext{
*Correspondence: aso.rasheed@univsul.edu.iq

${ }^{1}$ Medical College, Sulaimany University, Sulaimany, Iraq

Full list of author information is available at the end of the article
}

cause dilatation and relaxation of the smooth muscles of the urinary tract and may help spontaneous stone passage throughout the urinary tract [3]. This usually occurs during the second and third trimesters since progressive dilation of the ureter allows the asymptomatic actual ureteric stone to migrate downward, embed in the middle or lower ureter and become symptomatic [4]. Physiological hydronephrosis also poses significant management challenges in a clinical trial of ureteric stones, and therefore, stone documentation is mandated. Physiological 
hydronephrosis is much more common on the right side since it is multifactorial [5]. The incidence of physiologic hydronephrosis is as high as $90 \%$ on the right side and $67 \%$ on the left side during pregnancy. Although this typically resolves within one month after delivery, its laterality throughout pregnancy has shown no association with factors such as urinary tract problems or prior pregnancies [6]. At the same time, ureteric stone carries risks for the pregnant woman and her foetus, and consequently, clinical intuition during decision making and the course of management is crucial. Preterm delivery, miscarriage, premature rupture of the membrane, and preeclampsia might be included among those risks [7]. The majority $(70-80 \%)$ of ureteric stones pass spontaneously by conservative treatment [8]. Close follow-up should be performed during conservative treatment; any clinical suspension or impending complication may take the urologist into the stage of active management to relieve obstruction and preserve renal function.

Objective: To evaluate the safety and efficacy of ureteroscopy with auxiliary procedures as a primary treatment for pregnant women with symptomatic ureteric stones who have failed conservative management.

\section{Methods}

This was a prospective cohort study. We evaluated 26 pregnant women who presented to our emergency department with an initial diagnosis of symptomatic ureteric stone and hydronephrosis that did not resolve despite conservative treatment during the follow-up period between June 2017 and November 2019. Written informed consent was obtained from all the participants to participate in the study. All patients were offered conservative treatment (such as intravenous fluid replacement and analgesics) as a primary modality of treatment. Those who had failed medical therapy or had an absolute indication for active management at the time of presentation were included in the study. The inclusion criteria were as follows: pregnant women who were 16 years and above, failure to respond to conservative management for ureteric stones, all stages of pregnancy, all symptomatic ureteric stones, urosepsis, severe hydroureteronephrosis, renal impairment in a solitary kidney or bilateral disease; and persistent, intractable pain. The exclusion criteria were spontaneous stone passage during conservative measurement, asymptomatic ureteric stones, colic due to renal stones, and physiological hydronephrosis and comorbidities such as eclampsia.

All patients had a full history and a clinical examination to record their gestation trimesters, admission symptoms, diagnostic methods, degree of hydronephrosis, history of ureteric stone or urological interventions, and sizes and locations of stones. Baseline laboratory tests included general urinalysis, urine culture, complete blood count, serum creatinine, blood urea nitrogen (BUN), and C-reactive protein. Ultrasound was used as a primary diagnostic modality for stone documentation. Preoperative patients' data were recorded. All patients were offered spinal anesthesia, a prophylactic antibiotic with a 3rd-generation cephalosporin, and each patient was positioned in a low lithotomy position with slight tilting to the left. The proposed operation, and the modality of treatment was decided intra-operatively to relieve the obstruction, followed by intraoperative decisions for further endourological intervention (stone factor, ureteral status, patient factor, and renal factor). Accordingly, the patients were offered semi-rigid $8 \mathrm{Fr}$ tip ureterorenoscopy (Karl Storz Endoscopy, Tuttlingen, Germany) and flexible ureterorenoscopy 7.5 Fr (Storz Flex-X2S, Tuttlingen, Germany) without an access sheath, using a Holmium-Yttrium-Aluminium-Garnet (Ho: YAG) laser (CALLCULASE II SCB, Karl Storz, Germany) as a primary lithotripter. The frequency was adjusted to $6-8 \mathrm{~Hz}$ and the energy to $0.8-1.2 \mathrm{~J} /$ pulse. A Glide wire (Terumo, 0.035 inches) was used as a primary working guidewire following the guidewire technique for accessing the ureter. Stones larger than $2 \mathrm{~mm}$ were extracted using a stone extracting device. All patients underwent 4.7- $6 \mathrm{Fr}, 26 \mathrm{~cm}$, double J-stent insertion. The double JJ was removed after 3-4 weeks.

Accordingly, there were 3 patient groups: group A had a semi-rigid ureteroscope $8 \mathrm{~F}$ tip with laser lithotripsy, Group B received 7.5 Fr flexible ureterorenoscopy (URS) with laser lithotripsy and a $3.0 \mathrm{Fr}$ stone basket (zero-tip nitinol stone-retrieval basket), which was used as a stone-extracting device in both groups. Group $\mathrm{C}$ underwent semi-rigid ureteroscopy and stone extraction using a PULSELITH-triponge grasper without laser lithotripsy. Outcomes study were made between the three groups in terms of safety and efficiency.

Intraoperative and postoperative complications were classified according to the Clavien-Dindo classification system [9]. Each patient was monitored for intraoperative ureteral injury by using the following Modified Satava Classification: Grade 1: ureteral injury without sequelae; Grade 2A: intraoperative treatment with endoscopic surgery; Grade 2B: endoscopic retreatment in the second operation; and Grade 3: incidents requiring exploration with open surgery or laparoscopic intervention [10]. The patients were sent for an abdominal ultrasound on the 1st postoperative day for rechecking of the JJ stent, for stone-free status or residual stones and for any perirenal collection. Postoperative renal function was assessed by serum creatinine level. 
All patients were followed until the end of pregnancy and after delivery to ensure maternal and foetal well-being.

We defined stone-free status as the absence of fragments of more than $1 \mathrm{~mm}$ in diameter in the ureter, which are too small to be extracted with a basket or grasper by ureteroscopy inspection.

The method was considered successful if no stone was noticed in the ureter, the ureter was patent, a double J stent was inserted, and the patient's symptoms resolved.

Efficacy was defined by the stone clearance rate and the need for additional procedures such as ureteric stenting. Process and recovery outcomes were measured by the length of hospital stay and analgesic requirements. Intraoperative postoperative patients and operative findings were collected for statistical analysis using the Statistical Package for Social Sciences version 22.0 software. The chi-square test and one-sample T-test were used to calculate the P-value. A P-value of $<0.05$ was chosen for statistically significant data.

\section{Results}

Twenty-six pregnant women had symptomatic ureteral stones, with a mean age and standard deviation (SD) of $27.58 \pm 4.61$ years and a mean gestational age of $28.38 \pm 6.34$ SD weeks. Of the total of 26 stones, 20 $(76.9 \%)$ were in the lower ureter, zero were in the middle one-third of the ureter, and 6 (23.1\%) were in the upper one-third of the ureter.

Left-sided ureteric stones were encountered in 7 (26.9\%) patients, and right-sided stones were encountered in $19(73.1 \%)$ patients. The size of the stones ranged from 6 to $12 \mathrm{~mm}$ (mean: $7.38 \mathrm{~mm}$ ), as estimated by abdominal ultrasound. The main presenting symptom was flank pain in 22 patients $(84.61 \%)$ and nausea with vomiting in 4 patients (15.38\%). The endourological intervention achieved persistent pain in 22 patients $(84.61 \%)$, severe hydronephrosis in three patients (11.53\%), and progressive hydronephrosis in 1 patient (3.8\%). See Table 1.

The first group of 10 patients managed by flexible ureterorenoscopy with laser lithotripter and double J-stent insertion had a stone-free rate of $100 \%$. The mean operative time was $49.2 \pm 5.84$ SD minutes, and the mean postoperative serum creatinine was $0.93 \pm 0.188 \mathrm{SD} \mathrm{mg} / \mathrm{dl}$, with intraoperative grade 1 (Satava classification) ureteral injury, postoperative complication grade 1 (ClavienDindo) in $70 \%$ of patients and grade 2 in $30 \%$ of patients. The mean duration of postoperative hospitalization was $2.4 \pm 0.516 \mathrm{SD}$; no patients required a secondary operation apart from double J-stent removal under local anesthesia with flexible cystoscopy after 3-4 weeks.

In the second group of 11 patients managed by semirigid ureterorenoscopy, the stone-free rate was $72.7 \%$, the

\begin{tabular}{|c|c|}
\hline Variable & Value \\
\hline Age (years) & $18-34(27.58 \pm 4.61)$ \\
\hline Gestational age (weeks) & $12-36(28.38 \pm 6.34)$ \\
\hline \multicolumn{2}{|l|}{ Clinical presentation (\%) } \\
\hline Flank pain & $22(84.6 \%)$ \\
\hline Nausea and vomiting & $4(15.4 \%)$ \\
\hline \multicolumn{2}{|l|}{ Side of the stone (\%) } \\
\hline Right & $19(73.1 \%)$ \\
\hline Left & $7(26.9 \%)$ \\
\hline History of stone disease $\%$ & $9(34.6 \%)$ \\
\hline \multicolumn{2}{|l|}{ Size of the stone $(\mathrm{mm})$} \\
\hline Range & $6-12$ \\
\hline Mean & 7.38 \\
\hline \multicolumn{2}{|l|}{ Diagnostic tool (\%) } \\
\hline Ultrasound & $19(73.1 \%)$ \\
\hline Ureterorenoscopy & $7(26.9 \%)$ \\
\hline \multicolumn{2}{|l|}{ Site of the stone (\%) } \\
\hline Lower ureter & $20(76.9 \%)$ \\
\hline Middle ureter & $0(0.0 \%)$ \\
\hline Upper ureter & $6(23.1 \%)$ \\
\hline \multicolumn{2}{|l|}{ Serum creatinine (mg/dl) } \\
\hline Range & $0.5-1.3$ \\
\hline Mean & 0.81 \\
\hline \multicolumn{2}{|l|}{ Degree of hydronephrosis (\%) } \\
\hline Mild & $10(38.46 \%)$ \\
\hline Moderate & $13(50 \%)$ \\
\hline Severe & $3(11.53 \%)$ \\
\hline
\end{tabular}

mean operative time was $31.73 \pm 3.19$, the mean postoperative serum creatinine was $0.78 \pm 0.13 \mathrm{mg} / \mathrm{dl}$, with intraoperative G1 (Satava classification) ureteral injury in 2 patients, postoperative complication Grade 1 (ClavienDindo) in $72.7 \%$ of patients and Grade 2 complications in $27.3 \%$ of patients. The mean postoperative hospitalization was $2.73 \pm 1.009$ Eight stone-free patients had double $\mathrm{J}$ stents that were removed under local anesthesia with flexible cystoscopy after 3-4 weeks. Three patients with upper ureteric stones (residual stones and stone fragments pushed backed during lithotripsy) were kept on double J stents until delivery. Then, they underwent a secondary (delayed) operation with flexible ureterorenoscopy within 6-8 weeks postdelivery, and the stone-free rate was $100 \%$.

The third group of 5 patients were managed by using semi-rigid ureterorenoscopy with a triponge grasper without mandating lithotripsy. The stone-free rate was $100 \%$, the mean operative time was $22.4 \pm 1.14$ SD minutes, and the mean postoperative serum creatinine was $0.8 \pm 1.158$. Intraoperative G1 ureteral injury occurred 
in three patients. Grade 1 postoperative complications occurred in $100 \%$ of patients, and there were no Grade 2 complications. The mean postoperative hospitalization was $1.60 \pm 0.548$ days. See Tables 2 and 3 .

\section{Discussion}

During pregnancy, the accurate diagnosis of ureteric stones is masked by physiological changes. Clinical signs and symptoms, microscopic haematuria, and greyscale ultrasound can be used as primary modalities for diagnosis. The most common clinical presentation of ureteric stones in pregnant patients is loin pain. Andreoiu et al. in 2009 reported loin pain in $96.5 \%$ of 144 confirmed cases [11]. In our study, loin pain occurred in 22 patients $(84.6 \%)$ in 26 confirmed cases; however, the clinical presentation of ureteric stones in pregnancy became less predictable for diagnosis, since loin pain and microscopic haematuria may occur as part of the ordinary course of pregnancy.

More stones on the right side are diagnosed because the physiologic hydronephrosis that is diagnosed by ultrasound more often on the right side raises the suspicion of ureteric stones and may miss left-sided stones because of bowel gas from the sigmoid colon.

In the acute presentation, grayscale ultrasound may miss ureteric stones, with a high false-negative rate but high sensitivity when there is clinical suspicion or radiological signs such as hydronephrosis exist. The sensitivity and specificity of ultrasonography for renal stone disease are $59 \%-78 \%$ and $100 \%$, respectively [12].

Lifshitz DA et al. retrospectively analyzed ten symptomatic pregnant women who had ureteric stones that required URS in 2000. The mean patient age was 23 (range 17-31) years. Ultrasound scanning was performed in all patients and showed a low sensitivity $(28.5 \%)$ when compared with intraoperative findings [13].

In our study, we used ultrasound as the primary modality for diagnosis and applied it to all patients. Stones yielded a positive result in $73.1 \%$ of patients, and $26.9 \%$ of patients with high clinical suspicion of ureteric stones were diagnosed during ureteroscopy.

Few ultrasonic parameters can help, such as using a transvaginal probe, monitoring the ureteric jet, and measuring the resistive index [14], and using colour Doppler for the assessment of the ureteral jet may assist in the diagnosis of ureteral obstruction [15]. Measuring the renal artery resistive index will facilitate the diagnosis of the obstruction [16].

Other modalities for diagnosis include MRI, lowdose CT scans $(<3.5 \mathrm{mSv})$, or ultralow-dose CT scans $(<1.9 \mathrm{mSv})$ [17]. The overall sensitivity of the diagnostic modalities of ultrasound, MRI, and CT for detecting

Table 2 Outcomes of Intraoperative and postoperative patient data in different groups

\begin{tabular}{|c|c|c|c|c|}
\hline Parameter & $\begin{array}{l}\text { Flexible URS + laser } \\
\text { lithotripsy } n: 10\end{array}$ & $\begin{array}{l}\text { Semi-rigid URS + laser } \\
\text { lithotripsy } n: 11\end{array}$ & $\begin{array}{l}\text { URS + triponge grasper } \\
\text { without lithotripsy } n: 5\end{array}$ & $p$-value \\
\hline Mean operative time (minutes) & $49.2 \pm 5.84$ & $31.73 \pm 3.19$ & $22.40 \pm 1.14$ & 0.998 \\
\hline Stone-free rate (\%) & $100 \%$ & $72.7 \%$ & $100 \%$ & 0.954 \\
\hline Mean postoperative serum creatinine (mg/dl) & $0.93 \pm 0.188$ & $0.78 \pm 0.13$ & $0.8 \pm 0.158$ & 0.872 \\
\hline Mean postoperative hospitalization (day) & $2.4 \pm 0.516$ & $2.73 \pm 1.009$ & $1.60 \pm 0.548$ & 0.978 \\
\hline Postoperative complication Clavien-Dindo-G1 & $70 \%$ & $72.7 \%$ & $100 \%$ & 0.993 \\
\hline Postoperative Clavien-Dindo complication-G2(fever) & $30 \%$ & $27.3 \%$ & $0 \%$ & 0.422 \\
\hline Mean gestational age (weeks) & $29.20 \pm 5.22$ & $26.73 \pm 7.68$ & $30.40 \pm 5.32$ & 0.997 \\
\hline Postoperative analgesia & $1(10 \%)$ & $2(18.18 \%)$ & $1(20 \%)$ & 0.043 \\
\hline
\end{tabular}

Table 3 Clavien-Dindo grades of the complications in different groups

\begin{tabular}{|c|c|c|c|c|}
\hline Clavien-Dindo grades & Complications & $\begin{array}{l}\text { Flexible URS + laser } \\
\text { lithotripter } n: 10\end{array}$ & $\begin{array}{l}\text { Semi-rigid URS + laser } \\
\text { lithotripter } n: 11\end{array}$ & $\begin{array}{l}\text { URS + triponge } \\
\text { stone extraction } \\
n: 5\end{array}$ \\
\hline \multirow[t]{6}{*}{ Grade 1} & Mild haematuria & $5(50 \%)$ & $7(63.6 \%)$ & $4(80 \%)$ \\
\hline & Stent-related symptoms & $9(90 \%)$ & $10(90.9 \%)$ & $3(60 \%)$ \\
\hline & Headache & $6(60 \%)$ & $8(72.7 \%)$ & $2(40 \%)$ \\
\hline & Nausea and vomiting & $8(80 \%)$ & $9(81.8 \%)$ & $4(80 \%)$ \\
\hline & Mild loin pain (fullness) & $7(70 \%)$ & $6(54.5 \%)$ & $2(40 \%)$ \\
\hline & Overall complication & $70 \%$ & $72.7 \%$ & $100 \%$ \\
\hline Grade 2 & Fever & $3(30 \%)$ & $3(27.3 \%)$ & $0(0 \%)$ \\
\hline
\end{tabular}


ureteric stones during pregnancy is $77 \%, 80 \%$, and $95.8 \%$, respectively [18].

In 2001, Watterson et al. performed a retrospective analysis of eight patients with a total of 10 symptomatic ureteral calculi. They underwent URS with laser management. Stone access and complete fragmentation were achieved in all patients. The overall procedural success rate was $91 \%$, with eight of nine calculi treated successfully (stone-free rate: 89\%) [19]. Latina et al. performed a systematic review of the literature from January 1990 to June 2011 to evaluate the clinical efficacy and safety of ureteroscopy as a primary treatment for pregnant women with symptomatic ureteric stones. A total of 239 abstracts were screened, and 15 studies that reported on 116 procedures were identified. The complete stone clearance rate was $86 \%$, with two major complications and seven minor complications [20]. A recent systematic review by Semins et al. clarified that 14 of 108 pregnant patients who underwent ureteroscopy stone treatment had an overall urological complication rate of $8.3 \%$, with no significant differences in ureteral injury and urinary tract infection compared with nonpregnant women [21].

The mean operative time was higher among the flexible URS group (49.2 \pm 5.84$)$ versus the semi-rigid URS group ( $31.73 \pm 3.19)$, which was explained by the fact that the handling and use of lithotripsy in a flexible device to target a stone is sensitive to respiratory movement, especially in patients under spinal anesthesia.

A retrospective study performed by Johnson EB et al. for assessing postoperative obstetrical complications in pregnant women who underwent ureteroscopy identified preterm labour in 2 of 46 patients at five institutions, with an overall obstetric complication rate of 4.3\% [22].

In our study, the gestational period showed no significant differences between the groups. There were no preterm labor cases or major obstetric complications because our primary decision was to relieve obstruction, and difficult circumstances were postponed for a delayed operation.

In our study, the overall stone-free rate was 23 out of $26(88.46 \%)$; the total success rate was $87 \%$, with no significant difference in the mean operative time when the semi-rigid ureteroscope was used. The overall postoperative complications were classified as Clavien-Dindo grade 1 in 20 patients (76.9\%), who had mild complications (loin pain, haematuria, nausea and vomiting, and stent-related symptoms) and as Clavien-Dindo grade 2 in 6 patients $(23.07 \%)$, who had a postoperative fever. Ureteral injury with a Satava classification of G1 (ureteral injury without sequelae) occurred in 6 patients (23.55\%).

Postoperative analgesia was used in 4 of 26 patients, which was significantly different between the groups and generally reflecting the success of endourology in pregnancy, which reduced the amount of analgesia needed after relieving the obstruction.

The American Urological Association (AUA) guidelines approve an initial conservative treatment with expected spontaneous stone passage [19]. Medical expulsive therapies (METs) such as alpha-blockers (tamsulosin) and calcium channel blockers (nifedipine) have no US Food and Drug Administration (FDA) approval for use during pregnancy [23].

Historically, the temporary modality has been considered the gold-standard management because it is less invasive and potentially useful in relieving obstruction and pain and maintaining optimal renal function $[24,25]$. Active urinary tract infection or urosepsis is an absolute contraindication for definitive stone treatment. In our study, during active management, none of the cases had urosepsis on presentation; the proposed operation was employed to alleviate obstruction with a JJ stent, and it was intraoperatively decided that definitive treatment would proceed based on stone, kidney, and patient factors.

A more recent retrospective study performed by Abedi AR et al. reported the safety and efficacy of URS and laser lithotripsy in 15 pregnant women who had ureteral stones, and there were no significant complications; however, the authors noted the limitations of the study, such as the retrospective nature and the small sample size [26]. Overall, these studies do not provide clear evidence supporting the safety of ureteroscopy and anesthesia during pregnancy, and the lack of fluoroscopic control requires advanced surgical techniques and surgeon experience. The limitation of our study was the limited number of cases. Further investigations should be performed, and our study can be used as a reference.

\section{Conclusion}

Ureteric stone in pregnancy requires a high index of suspicion for a prompt and correct diagnosis. Ureteroscopy: flexible or semirigid endoscopy combined with holmium: YAG laser lithotripsy or with stone extraction are a preferred modality for the definitive treatment of symptomatic ureteric stone in pregnancy.

\section{Supplementary Information}

The online version contains supplementary material available at https://doi. org/10.1186/s12301-021-00148-8.

Additional file 1. Patients row and statistical data in Excel sheet.

\section{Acknowledgements}

I would like to thank the staff and members of Sulaimanyah University Hospital for their kind assistant and support during working and research writing. 


\section{Authors' contributions}

AOR supervised and conducted the study. RYA collected and analyzed the data, reviewed the literature, and wrote the first draft collectively. All authors have read and approved the manuscript", and ensure that this is the case.

\section{Funding}

This research received no specific charity from any funding agency in the public or commercial, and no competing financial interests exist.

\section{Availability of data and materials}

All data generated or analyzed during this study are included in this published article (and its supplementary information files).

\section{Ethics approval and consent to participate:}

All subjects filled in an informed consent form and written informed consent was obtained from all the participants to participate in the study. Ethical review board committee approval was granted by the Medical College of the Sulaimanyah University- Iraq, with a number 2047, on 3rd of June 2017.

\section{Consent for publication}

Not applicable.

\section{Competing interest}

There is no conflict of interest.

\section{Author details}

${ }^{1}$ Medical College, Sulaimany University, Sulaimany, Iraq. ${ }^{2}$ Department of Urology, Sulaimany University Hospital, Sulaimany, Iraq.

Received: 12 June 2020 Accepted: 18 February 2021

Published online: 06 March 2021

\section{References}

1. Riley JM, Dudley AG, Semins MJ (2014) Nephrolithiasis and pregnancy: has the incidence been rising? J Endourol 28(3):383-386

2. Semins MJ, Matlaga BR (2013) Management of urolithiasis in pregnancy. Int J Women's Health 5:599

3. Sefa R, Cetin EH, Gurkan K, Metin K (2006) Are changes in urinary parameters during pregnancy clinically significant? Urol Res 34(4):244-248

4. Biyani CS, Joyce AD (2002) Urolithiasis in pregnancy. II: management. BJU Int. 89(8):819-823

5. Lewis DF, Robichaux AG, Jaekle RK, Marcum NG, Stedman CM (2003) Urolithiasis in pregnancy: diagnosis, management, and pregnancy outcome. Obstet Gynecol Surv 58(7):446-447

6. Peake SL, Roxburgh HB, Langlois SL (1983) Ultrasonic assessment of hydronephrosis of pregnancy. Radiology 146(1):167-170

7. Swartz MA, Lydon-Rochelle MT, Simon D, Wright JL, Porter MP (2007) Admission for nephrolithiasis in pregnancy and risk of adverse birth outcomes. Obstet Gynecol 109(5):1099-1104

8. Burgess KL, Gettman MT, Rangel LJ, Krambeck AE (2011) Diagnosis of urolithiasis and the rate of spontaneous passage during pregnancy. J Urol 186(6):2280-2284
9. Dindo D, Demartines N, Clavien P-A (2004) Classification of surgical complications: a new proposal with evaluation in a cohort of 6336 patients and results of a survey. Ann Surg 240(2):205

10. Tepeler A, Resorlu B, Sahin T, Sarikaya S, Bayindir M, Oguz U et al (2014) Categorization of intraoperative ureteroscopy complications using modified Satava classification system. World J Urol 32(1):131-136

11. Andreoiu M, MacMahon R (2009) Renal colic in pregnancy: lithiasis or physiological hydronephrosis? Urology 74(4):757-761

12. Viprakasit DP, Sawyer MD, Herrell SD, Miller NL (2012) Limitations of ultrasonography in the evaluation of urolithiasis: a correlation with computed tomography. J Endourol 26(3):209-213

13. Lifshitz DA, Lingeman JE (2002) Ureteroscopy as a first-line intervention for ureteral calculi in pregnancy. J Endourol 16(1):19-22

14. Laing FC, Benson CB, DiSalvo DN, Brown DL, Frates MC, Loughlin KR (1994) Distal ureteral calculi: detection with the vaginal US. Radiology 192(2):545-548

15. Burke BJ, Washowich TL (1998) Ureteral jets in normal second-and thirdtrimester pregnancy. J Clin Ultrasound 26(9):423-426

16. Shokeir AA, Mahran MR, Abdulmaaboud M (2000) Renal colic in pregnant women: role of a renal resistive index. Urology 55(3):344-347

17. Rob S, Bryant T, Wilson I, Somani BK (2017) Ultra-low-dose, low-dose, and standard-dose CT of the kidney, ureters, and bladder: is there a difference? Results from a systematic review of the literature. Clin Radiol 72(1):11-15

18. White WM, Johnson EB, Zite NB, Beddies J, Krambeck AE, Hyams E et al (2013) Predictive value of current imaging modalities for the detection of urolithiasis during pregnancy: a multicenter, longitudinal study. J Urol 189(3):931-934

19. Watterson JD, Girvan AR, Beiko DT, Nott L, Wollin TA, Razvi H et al (2002) Ureteroscopy and holmium: YAG laser lithotripsy: an emerging definitive management strategy for symptomatic ureteral calculi in pregnancy. Urology 60(3):383-387

20. Laing KA, Lam TBL, McClinton S, Cohen NP, Traxer O, Somani BK (2012) Outcomes of ureteroscopy for stone disease in pregnancy: results from a systematic review of the literature. Urol Int 89(4):380-386

21. Semins MJ, Trock BJ, Matlaga BR (2009) The safety of ureteroscopy during pregnancy: a systematic review and meta-analysis. J Urol 181(1):139-143

22. Johnson EB, Krambeck AE, White WM, Hyams E, Beddies J, Marien T et al (2012) Obstetric complications of ureteroscopy during pregnancy. J Urol 188(1):151-154

23. Lloyd GL, Lim A, Hamoui N, Nakada SY, Kielb SJ (2016) The use of medical expulsive therapy during pregnancy: a worldwide perspective among experts. J Endourol 30(3):354-358

24. Assimos D, Krambeck A, Miller NL, Monga M, Murad MH, Nelson CP et al (2016) Surgical management of stones: American urological association/ endourological society guideline. PART I J Urol 196(4):1153-1160

25. Choi Cl, Yu YD, Park DS (2016) Ureteral stent insertion in the management of renal colic during pregnancy. Chonnam Med J 52(2):123-127

26. Abedi AR, Allameh F, Razzaghi MR, Fadavi B, Qashqai H, Najafi S et al (2017) The efficacy and safety of laser lithotripsy in pregnancy. J lasers Med Sci 8(2):84 